\title{
The system of speech enhancement algorithm for blind source separation based on FastICA
}

\author{
Minsan Zhang \\ Hunan Railway Professional Technology College, Zhuzhou, 412001, China
}

Keywords: speech enhancement algorithm, blind source separation, FastICA.

\begin{abstract}
A speech based on FastICA blind source separation algorithm. It has been transplanted to a speech enhancement system whose core component is DSP chip. This system enhances speech signals in real time. It samples sounds signals in the air using a microphone array and uses its embedded speech enhancement algorithm separates speech source signals and noise source signals, in order to suppress noise source signals including inference speech signals. There is also a further step that suppressing residual background noise after separation.
\end{abstract}

\section{Introduction}

Speech enhancement is an important source of system to deal with noise is active noise, noise emitted from a point source, especially with channel noise (voice signal of the target speech signal outside). Because most of the existing noise algorithm parameters, in accordance with the noise outside voice and with channel noise statistical properties of noise suppression, apparently based speech enhancement algorithm above systems are incapable of action on the same channel noise. To this end, we will design a pair of active noise, mainly with the channel noise has better inhibition effect of speech enhancement system as the target. We use the Fast ICA algorithm is a blind source separation algorithm of this system. The successful application of Fast ICA algorithm in speech enhancement and it is ported to embedded system, we want to solve problems.

\section{The principle of the system}

The working principle of the calorimeter. The working principle of the calorimeter is: oxygen bomb weighed analysis of coal samples with excess oxygen in the charge of the full combustion, the heat released by using quantitative water absorption, according to produce sample combustion calor imetry system before and after the temperature rise, will be measured by the temperature sensor PT1000 change and the electrical signal causes the corresponding collection, temperature changes and to point the fiery, calorimetric system with external environments such as the heat exchange of heat caused by correction is carried out through the $\mathrm{A} / \mathrm{D}$ conversion, digital filter method is used in certain filtering processing, and using the empirical formula for the revised.

The hardware of the system overall design. The hardware design of the system mainly consists of two parts: the card and the man-machine interface board control card. Completed the main control card motherboard temper-ature acquisition, ignition control and ignition judgment function. The man-machine interface card is mainly to complete data storage, liquid crystal display and printer output control etc. The hardware plat for adopts high performance to price ratio of the programmable logic deviceATF1508AS build, between the board and the board through the SPI bus communication.

\section{The design of hardware circuit unit}

Design of temperature acquisition circuit. The measurement of the quantity of heat meter accuracy directly reflected in the temperature measurement accuracy, so whether can the temperature rise for accurate measurement of the accuracy of the instrument is very big impact, in the acquisition of inner and outer barrel temperature, this design uses the temperature sensor PT1000 accuracy of 0.0001 DEG C temperature acquisition, the schematic diagram shown in Fig.1 


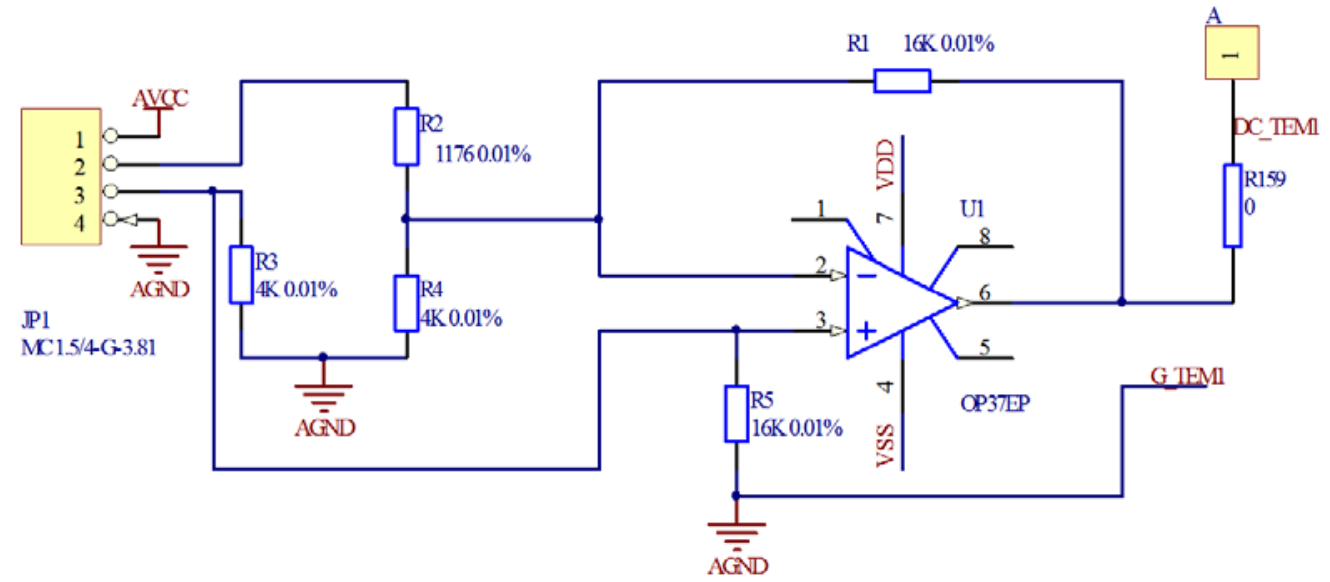

Fig.1 The temperature acquisition module circuit diagram

Design of operational amplifier inverting input for potential, potential input phase, potential output is DC $+5 \mathrm{~V}$ power supply, AVCC, IP1 as the PT1000 temperature sensor interface. By the circuit schematic diagram and the operational amplifier "virtual short circuit" and "virtual circuit" that:

$$
\begin{aligned}
& \frac{5-V-}{1176}=\frac{V-}{4000}+\frac{V--V o}{16000} \\
& V+=V- \\
& R_{t}=\frac{94080000-3200 * 1176 V o}{80000+1176 V o} \\
& V+=\frac{5}{R_{t}+(4000 / / 16000)} *(4000 / / 16000)
\end{aligned}
$$

The $(4000 / / 16000)$ is expressed in parallel resistance.

Design of A/D conversion circuit. Measurement of inner barrel and outer barrel temperature, two temperature acquisition channel, with four channel 24 bit A/D converter A/D conversion on the core ADS1253 collected signal. Using ADR444BRZ as the voltage reference chip, provides reference voltage A/D sampling. The principle diagram of Fig.2 and Fig.3

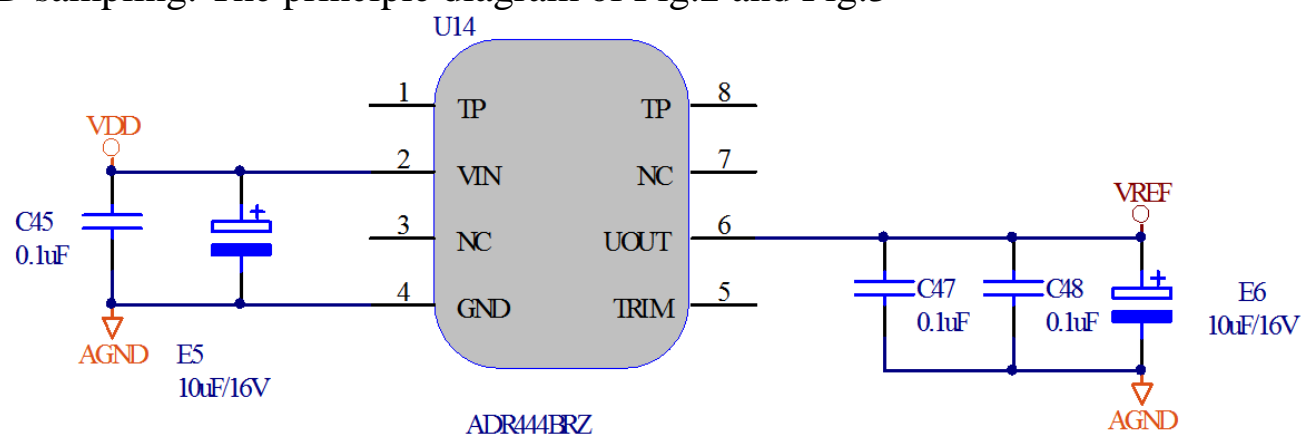

Fig.2 A/D conversion circuit of reference voltage circuit

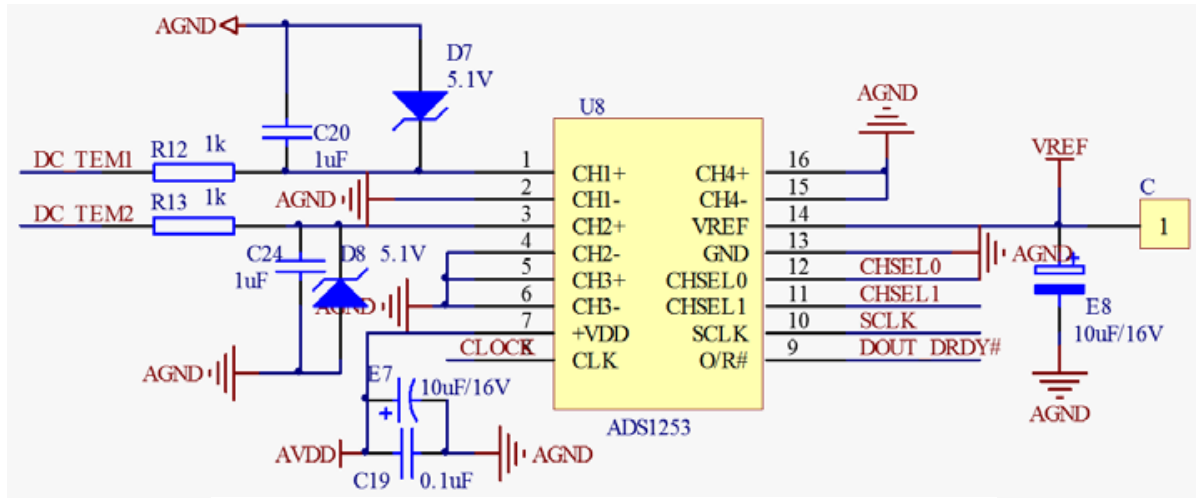

Fig.3 A/D conversion circuit principle diagram 
Design of clock circuit. According to the system to achieve real-time requirements, design and selection of serial clock chip PCF8563, and MCU less connection, greatly saves system resources of mcu. PCF8563 and MCU interface circuit as shown in Fig.4. And the part of the circuit also add the power-off protection function, in the main power supply system of unexpected power, namely Vcc 0V, D1 cutoff,TL5186 standby power to continue to supply PCF8563 through D3, ensure the normal work of the clock chip.

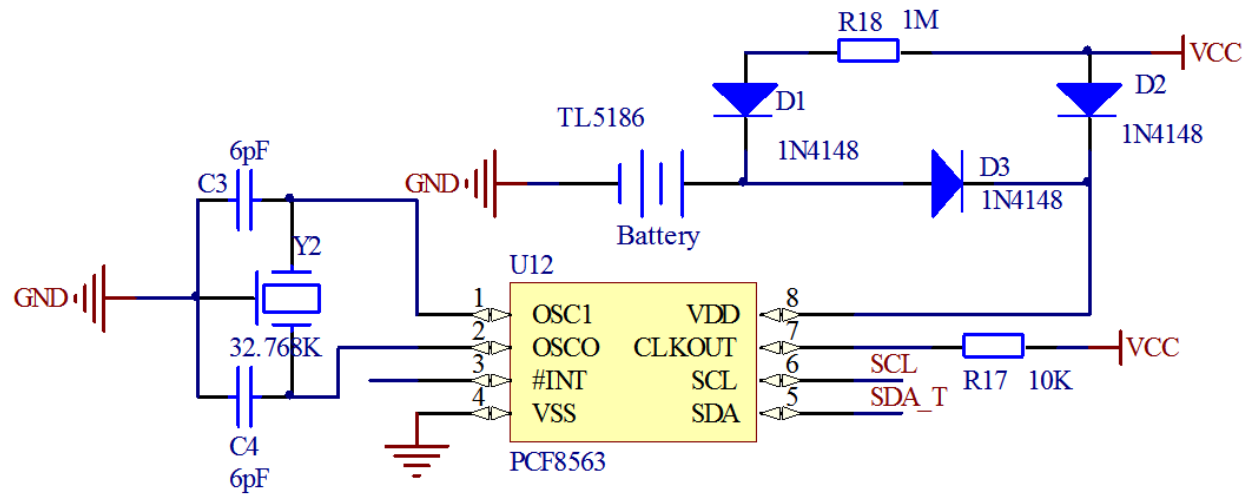

Fig.4 clock circuit

\section{Temperature sampling process}

The initial sampling. In order to calculate the cooling correction, need to know the cooling speed of the inner cylinder ignition. Therefore, to set an initial stage is during the test, with the average cooling rate as the cooling rate at ignition. The inner cylinder temperature is uniform, accurate measurement of a temperature value. The quasi to 0.001 DEG C, denoted as t0_Fri, at the same time start timing. After 5 minutes, to accurately measure a temperature, $\mathrm{t} 0$. Then the measured and calculated V0 (ignition when the cooling speed of inner cylinder).

The main phase sampling. After the ignition, into the main stage test. If the temperature rises sharply after 45s, it shows that the ignition success. Otherwise the ignition failure. In the process of temperature rise, temperature rise curve gradually stabilized, should try to control the stability of the environmental temperature, caused by the decrease of temperature change due to temperature rise error, thus affecting the test results.

Stage sampling. Barrel temperature appears when decreased, test in the end. From the first drop in temperature point start time, 5 minutes later, accurate measuring cylinder temperature taking, denoted as TN. Stop stirring, end of test. Calculation of cooling rate, cooling rate and cooling rate on behalf of the end, as the calculation of cooling correction value for. At the end of the inner cylinder barrel, outward heat dissipation, the temperature drops unceasingly, therefore, at the end of the cooling rate is a positive. Set the stage, is to calculate average cooling rate at the end of.

Tab. 1 Test Data Sheet heat

\begin{tabular}{|c|c|c|c|c|c|c|c|}
\hline $\begin{array}{l}\text { Sample } \\
\text { weight (g) }\end{array}$ & $\begin{array}{l}\text { The main period of } \\
\text { time (min) }\end{array}$ & $\begin{array}{l}\text { Calorific } \\
\text { value (J/g) }\end{array}$ & $\begin{array}{c}\text { The } \\
\text { average heat } \\
\text { (J/g) }\end{array}$ & $\begin{array}{l}\text { The relative standard } \\
\text { deviation (\%) }\end{array}$ & $\begin{array}{c}\text { Range } \\
(\mathrm{J} / \mathrm{g})\end{array}$ & $\begin{array}{l}\text { Value } \\
(\mathrm{J} / \mathrm{g})\end{array}$ & $\begin{array}{l}\text { Compliance with } \\
\text { national standards }\end{array}$ \\
\hline 0.6908 & 9 & 26434.67 & \multirow{10}{*}{26445.54} & \multirow{10}{*}{0.09} & \multirow{10}{*}{53.17} & \multirow{10}{*}{26457} & \multirow{10}{*}{ yes } \\
\hline 0.9940 & 9 & 26457.91 & & & & & \\
\hline 1.3016 & 9 & 26459.79 & & & & & \\
\hline 1.2794 & 9 & 26463.60 & & & & & \\
\hline 0.7441 & 9 & 26409.89 & & & & & \\
\hline 0.7624 & 9 & 26431.67 & & & & & \\
\hline 1.0021 & 9 & 26457.69 & & & & & \\
\hline 1.3015 & 9 & 26459.79 & & & & & \\
\hline 1.2487 & 9 & 26461.10 & & & & & \\
\hline 0.7011 & 9 & 26419.33 & & & & & \\
\hline
\end{tabular}




\section{Test and analysis of test data}

The man-machine interface control board to control the main control board. The man-machine interface to test the transmission main control board test parameter, start command. The main control board completed the initial, stage and the last stage of sampling and control work, the calculation results of testdata, return the real-time temperature, test status to the human-computer interface control board. Through the test. The test measured data shown in the table below: (the decimal part test data were "four to six homes in five pairs".)

\section{Conclusion}

This design in strict accordance with the GB (T) 2013-2003 standard, using high precision temperature sensor PT1000 temperature acquisition, has stability, high after measurement, heat capacity and heat stability of the main period of time, relative standard deviation, range are in line with national standards. In the production of coal, electric power, petroleum chemical industry, cement industry and scientific research and the application prospect of good.

\section{The main references}

[1] Li Yinghua. Analysis of coal application technology guide [M]. Beijing: Standards Press of China. 2009 Chinese.

[2] Hu Biao, Meng Zhiqiang, Hu Yakuza. PCI bus technology in the calorimetersystem on the application of [J]. Instrument technique and sensor 2008 (40-4306).

[3] Hu Biao. Research and design of fast calorimeters. Hunan University master's degree thesis.2008 years.

[4] Single Yong, Xu Guangli. Application [J]. Shandong coal technology of intelligent automatic calorimeter. 2009 (01): 59-60.

[5] Cui Guangrun Zhong, Li Zhongyuan, ignition microcomputer calorimetrycontrol method of measured value influence of calorific capacity [J]. Journal of Zhoukou Normal University. 2003 (02):26-28

[6] Li Shulong, Yang Haisheng. The influence of the environment on the testing process of automatic calorimeter in coal quality analysis [J].technology. 2012 (11)

[7] Jiang Wei, Zhao Shujun, Xu hang. [J]. modern electronics technology. The design of isothermal automatic calorimeter based on S3C2410. 2010 (15)

[8] Lu Yang. Han Jianghong et al. The temperature compensation method of platinum resistance temperature measurement system [J]. Chinese Journal of scientific instrument. 2000 (03)

[9] Jiang Wei. The design of isothermal automatic calorimeter based on S3C2410. 2010. Zhengzhou University Master Thesis

[10] Li Ping. Research and design of isothermal automatic calorimeter based on MCU. Zhengzhou University master thesis. 2011. 